VOL. 44 (1991) [279-283]

\title{
UNIFORM PARTITION AND THE BEST LEAST-SQUARES PIECEWISE POLYNOMIAL APPROXIMATION
}

\author{
Franģois Dubeau
}

It is shown that the best least-squares piecewise $n$ degree polynomial approximation of $x^{n+1}$ over $[a, b]$ is obtained for a uniform partition. Moreover the approximation is continuous for $n$ odd and discontinuous, with equal stepsizes at the nodes, for $n$ even.

The problem considered here has been introduced by Stone [12] for $n=1$. In this paper Stone has considered the least-squares continuous piecewise linear approximation of a function $f(\cdot)$ over $[a, b]$. For a quadratic function $f(x)=p x^{2}+q x+r$, or essentially for $f(x)=x^{2}$, his result states that the optimal solution is obtained for the uniform partition and the global solution is given by the solution of a least-squares problem on each subinterval. For a general function $f(\cdot)$ he has proposed an iterative method for solving the necessary optimality conditions. The problem for $n=1$ has also been considered by Ream [10], Bellman [2], Gluss [6], Cantoni [3], Tomeck [13] and others. It is also related to the polygonal approximation of data for computer vision, graphics and image processing (see [7], [8] and [9]).

Let

$$
\prod_{N}=\left\{\Delta=\left\{x_{i}\right\}_{i=0}^{N} \mid a=x_{0}<\ldots<x_{i}<\ldots<x_{N}=b\right\}
$$

be the set of all partitions $\Delta$ of $[a, b]$ into exactly $N$ intervals. Let $\mathcal{P}^{n}\left[x_{i-1}, x_{i}\right]$ be the set of all polynomials of degree at most $n$ defined over $\left[x_{i-1}, x_{i}\right]$ and let

$$
\mathcal{P}_{N}^{n}=\prod_{i=1}^{N} \mathcal{P}^{n}\left[x_{i-1}, x_{i}\right]
$$

The object of this note is to show that the minimum of

$$
F(\triangle, \vec{p})=\sum_{i=1}^{N} \int_{x_{i-1}}^{x_{i}}\left(x^{n+1}-p_{i}(x)\right)^{2} d x
$$

Received 23 November 1990

This research has been supported by the Department of the National Defence of Canada and by "le Ministère de l'Education du Québec".

Copyright Clearance Centre, Inc. Serial-fee code: 0004-9729/91 \$A2.00+0.00. 
subject to $\Delta \in \Pi_{N}$ and $\vec{p}=\left(p_{1}(\cdot), \ldots, p_{N}(\cdot)\right) \in \mathcal{P}_{N}^{n}$, is obtained for the uniform partition $\triangle^{*}=\left\{x_{i}^{*}=a+i(b-a) / N\right\}_{i=0}^{N}$. Moreover the optimal $p_{i}^{*}(\cdot)$ 's form a continuous approximation for $n$ odd or a discontinuous approximation with equal stepsizes at the nodes for $n$ even. The optimal $\vec{p}^{*}=\left(p_{1}^{*}(\cdot), \ldots, p_{N}^{*}(\cdot)\right)$ is called the best least-squares piecewise $n$ degree polynomial approximation of $x^{n+1}$ over $[a, b]$.

The necessary conditions for the optimality of $p$ and $\Delta$ are:

(A)

$$
\int_{x_{i-1}}^{x_{i}}\left(x^{n+1}-p_{i}(x)\right) q(x) d x=0 \text { for all } q(\cdot) \in \mathcal{P}^{n}\left[x_{i-1}, x_{i}\right] \text { and } i=1, \ldots, N
$$

(B)

$\left[x_{i}^{n+1}-p_{i}\left(x_{i}\right)\right]^{2}-\left[x_{i}^{n+1}-p_{i+1}\left(x_{i}\right)\right]^{2}=0$ for all $i=1, \ldots, N-1$.

Before considering (A) and (B), let us recall some properties of the Legendre polynomials $\hat{\pi}_{\ell}(\cdot)(\ell=0, \ldots, k)$ defined on $[-1,1]$. They form an orthogonal basis of $\mathcal{P}^{k}[-1,1]$ with respect to the usual scalar product

$$
(\widehat{p}(\cdot), \widehat{q}(\cdot))=\int_{-1}^{1} \widehat{p}(\xi) \widehat{q}(\xi) d \xi .
$$

To obtain an orthogonal basis for $\mathcal{P}^{k}\left[x_{i-1}, x_{i}\right]$ we consider the transformation

where

$$
\begin{gathered}
x \longrightarrow \xi=T_{i}(x):\left[x_{i-1}, x_{i}\right] \longrightarrow[-1,1] \\
T_{i}(x)=\frac{\left(x-x_{i-1}\right)-\left(x_{i}-x\right)}{\left(x_{i}-x_{i-1}\right)},
\end{gathered}
$$

and the polynomials $\pi_{\ell, i}(\cdot)=\hat{\pi}_{\ell} \circ T_{i}(\cdot)(\ell=0, \ldots, k)$. It follows that the polynomials $\pi_{\ell, i}(\cdot)(\ell=0, \ldots, k)$ form an orthogonal basis of $\mathcal{P}^{k}\left[\boldsymbol{x}_{i-1}, \boldsymbol{x}_{\boldsymbol{i}}\right]$ with respect to the usual scalar product

$$
(p(\cdot), q(\cdot))=\int_{x_{i-1}}^{x_{i}} p(x) q(x) d x .
$$

The main properties of the polynomials $\hat{\pi}_{\ell}(\cdot)$ and $\pi_{\ell, i}(\cdot)$ are summarised in the table on the next page (see [4] or [11, pp.126-127]).

Using the orthogonal basis $\left\{\pi_{\ell, i}(\cdot) \mid i=0, \ldots, n+1\right\}$ for $\mathcal{P}^{n+1}\left[x_{i-1}, x_{i}\right]$, we can write $x^{n+1}=\sum_{\ell=0}^{n+1} \alpha_{\ell, i} \pi_{\ell, i}(x)$ where

$$
\alpha_{\ell, i}=\int_{x_{i-1}}^{x_{i}} x^{n+1} \pi_{\ell, i}(x) d x / \int_{x_{i-1}}^{x_{i}} \pi_{\ell, i}^{2}(x) d x
$$




\begin{tabular}{|c||c|c|}
\hline & $\widehat{\pi}_{\ell}(\cdot)$ & $\pi_{\ell, i}(\cdot)$ \\
\hline 1. & $\hat{\pi}_{\ell}(-1)=(-1)^{2}$ and $\hat{\pi}_{\ell}(1)=1$ & $\pi_{\ell, i}\left(x_{i-1}\right)=(-1)^{L}$ and $\pi_{\ell, i}\left(x_{i}\right)=1$ \\
\hline 2. & $\int_{-1}^{1} \xi^{\ell} \hat{\pi}_{k}(\xi) d \xi=0$ & $\int_{x_{i-1}}^{x_{i}} x^{\ell} \pi_{\ell, i}(x) d x=0$ \\
& $(\ell=0, \ldots, k-1)$ & $(\ell=0, \ldots, k-1)$ \\
\hline 3. & $\int_{-1}^{1} \xi^{k} \hat{\pi}_{k}(\xi) d \xi=\frac{2^{k+1}(k !)^{2}}{(2 k+1) !}$ & $\int_{x_{i-1}}^{x_{i}} x^{k} \pi_{k, i}(x) d x=\frac{\left(x_{i}-x_{i-1}\right)^{k+1}(k !)^{2}}{(2 k+1) !}$ \\
\hline 4. & $\int_{-1}^{1} \hat{\pi}_{k}(\xi) d \xi=\frac{2}{2 k+1}$ & $\int_{x_{i-1}}^{x_{i}} \pi_{k, i}^{2}(x) d x=\frac{\left(x_{i}-x_{i-1}\right)}{2 k+1}$ \\
\hline 5. & $\int_{-1}^{1} \hat{\pi}_{k}(\xi) \hat{\pi}_{\ell}(\xi) d \xi=0$ for $k \neq \ell$ & $\int_{x_{i-1}}^{x_{i}} \pi_{k, i}(x) \pi_{\ell, i}(x) d x=0$ for $k \neq \ell$ \\
\hline
\end{tabular}

TABLE. Basic properties of $\hat{\pi}_{\ell}(\cdot)$ and $\pi_{\ell, i}(\cdot)$.

for $\ell=0, \ldots, n+1$. In particular, using (3), we have

$$
\alpha_{n+1, i}=\frac{\left(x_{i}-x_{i-1}\right)^{n+1}[(n+1) !]^{2}}{(2 n+2) !}
$$

For a given partition $\Delta=\left\{x_{i}\right\}_{i=0}^{N}$, the solution of $(\mathrm{A})$ is

and

$$
\begin{gathered}
p_{i}^{*}(x)=\sum_{\ell=0}^{n} \alpha_{\ell, i} \pi_{\ell, i}(x) \\
x^{n+1}-p_{i}^{*}(x)=\alpha_{n+1, i} \pi_{n+1, i}(x) .
\end{gathered}
$$

It follows that

$$
\begin{aligned}
\int_{x_{i-1}}^{x_{i}}\left(x^{n+1}-p_{i}^{*}(x)\right)^{2} d x & =\int_{x_{i-1}}^{x_{i}}\left(\alpha_{n+1, i} \pi_{n+1, i}(x)\right)^{2} d x \\
& =\frac{\left(x_{i}-x_{i-1}\right)^{2 n+3}[(n+1) !]^{4}}{(2 n+3)[(2 n+2) !]^{2}}
\end{aligned}
$$

and

$$
F\left(\triangle, \vec{p}^{*}\right)=\frac{[(n+1) !]^{4}}{(2 n+3)[(2 n+2) !]^{2}} \sum_{i=1}^{N}\left(x_{i}-x_{i-1}\right)^{2 n+3} .
$$

But, from an inequality for weighted means (see [5] or [1]), we have

$$
\frac{1}{N} \sum_{i=1}^{N}\left(x_{i}-x_{i-1}\right)^{2 n+3} \geqslant\left(\frac{1}{N} \sum_{i=1}^{N}\left(x_{i}-x_{i-1}\right)\right)^{2 n+3}=\left(\frac{b-a}{N}\right)^{2 n+3}
$$

with a strict inequality if the $\left(x_{i}-x_{i-1}\right)$ are not all equal. Then

$$
F\left(\triangle, \vec{p}^{*}\right) \geqslant F\left(\Delta^{*}, \vec{p}^{*}\right)
$$


Hence $F(\triangle, \vec{p})$ is minimised for the uniform partition and the $\vec{p}_{i}(\cdot)$ 's are the best least-squares $n$ degree polynomal approximation of $x^{n+1}$ o over $\left[x_{i-1}, x_{i}\right]$.

Finally, for the uniform partition $\Delta^{*}$ we have

$$
x_{i}^{n+1}-p_{i}^{*}\left(x_{i}\right)=\alpha_{n+1, i} \pi_{n+1}(1)=\left(\frac{b-a}{N}\right)^{n+1} \frac{[(n+1) !]^{2}}{(2 n+2) !}
$$

and

$$
x_{i}^{n+1}-p_{i+1}^{*}\left(x_{i}\right)=\alpha_{n+1, i} \pi_{n+1}(-1)=(-1)^{n+1}\left(\frac{b-a}{N}\right)^{n+1} \frac{[(n+1) !]^{2}}{(2 n+2) !}
$$

Then

(i) the approximation is continuous for $n$ odd, and also $x_{i}^{n+1}-p_{i}^{*}\left(x_{i}\right)=$ $x_{i-1}^{n+1}-p_{i}^{*}\left(x_{i-1}\right)$

(ii) the approximation is discontinuous for $n$ even with equal stepsizes at the nodes:

$$
x_{i}^{n+1}-p_{i}^{*}\left(x_{i}\right)=p_{i+1}^{*}\left(x_{i}\right)-x_{i}^{n+1}
$$

and also

$$
x_{i}^{n+1}-p_{i}^{*}\left(x_{i}\right)=-\left(x_{i-1}^{n+1}-p_{i}^{*}\left(x_{i-1}\right)\right)
$$

\section{REFERENCES}

[1] E.F. Bechenbach and R. Bellman, Inequalities (Springer-Verlag, Berlin, Heidelberg, New York, 1965).

[2] R. Bellman, 'On the approximation of curves by line segments using dynamic programming', Comm. ACM 4 (1961), 284.

[3] A. Cantoni, 'Optimal curve fitting with piecewise linear functions', IEEE Trans. Comput. (1971), 59-67.

[4] E.W. Cheney, Introduction to approximation theory (McGraw-Hill, New York, 1966).

[5] R. Cooper, 'Notes on certain inequalities: II', J. London. Math. Soc. 2 (1927), 159-163.

[6] B. Gluss, 'Further remarks on line segment curve-fitting using dynamic programming', Comm. ACM 5 (1962), 441-443.

[7] H. Imai and M. Iri, 'Computational-geometric methods for polygonal approximations of a curve', Comput. Vision Graphics Image Process 36 (1986), 31-41.

[8] Y. Kurozumi and W.A. Davis, 'Polygonal approximation by the minimax method', Comput. Graphics and Image Processing 19 (1982), 248-264.

[9] M.K. Leung and Y.-H. Yang, 'Dynamic strip algorithm in curve fitting', Comput. Vision Graphics Image Process. 51 (1990), 146-165.

[10] N. Ream, 'Note on: Approximation of curves by line segments', Math. Comp. 15 (1961), 418-419. 
[11] F. Scheid, Numerical analysis, Schaum's outline series (McGraw-Hill, New York, 1968).

[12] H. Stone, 'Approximation of curves by line segments', Math. Comp. 15 (1961), 40-47.

[13] I. Tomek, 'Two algorithms for piecewise-linear continuous approximation of functions of one variable', IEEE Trans. Comput. 23 (1974), 445-448.

Département de Mathématiques

Collège militaire royal de Saint-Jean

Saint-Jean-sur-Richelieu

Québec

Canada, JOJ 1 RO 